\title{
Superintegrable Calogero-type systems admit maximal number of Poisson structures
}

\author{
C. Gonera \\ Department of Field Theory, University of Lodz, Pomorska 149/153, \\ 90-236 Lodz Poland \\ and \\ Y. Nutku
}

Feza Gürsey Institute P. O. Box 6 Çengelköy, Istanbul 81220 Turkey

April 25, 2001

We present a general scheme for constructing the Poisson structure of superintegrable dynamical systems of which the rational Calogero-Moser system is the most interesting one. This dynamical system is $2 N$ dimensional with $2 N-1$ first integrals and our construction yields $2 N-1$ degenerate Poisson tensors that each admit $2(N-1)$ Casimirs. Our results are quite generally applicable to all super-integrable systems and form an alternative to the traditional bi-Hamiltonian approach.

\section{Introduction}

Completely integrable systems can be cast into Hamiltonian form in more than one way [1]. In fact multi-Hamiltonian structure can be regarded as an alternative statement of complete integrability. A Liouville-integrable dynamical system in $2 N$ dimensional phase space admits $N$ first integrals which enable us to construct action-angle variables and reduce the solution to $N$ quadratures. On the other hand there are super-integrable dynamical systems that admit $2 N-1$ first integrals so that the solution requires only one quadrature. It is perhaps worth underlining that the existence of such additional conserved Hamiltonians is a rather rare property of dynamical systems and in no way necessary for Liouville-integrability. An important class of such super-integrable systems are of Calogero-type [2] and its relativistic Ruijsenaars-Schneider generalizations [3], as well as the Winternitz system

\footnotetext{
${ }^{1}$ Supported by KBN 2 P03B 13416
} 
[4]. They admit a complete set, i.e. maximal number of Poisson structures but this aspect of super-integrable systems has not received any attention. In this paper we shall present a general method for the construction of $2 N-1$ compatible Poisson structures for $2 N$-dimensional super-integrable dynamical systems. It can be regarded as a kind of generalization of Nambu mechanics [5] and its extensions to integrable dynamical systems with three degrees of freedom [6].

We shall present constructive proof of the complete Poisson structure of super-integrable systems. To make our construction explicit, in what follows we shall concentrate only on the rational Calogero-Moser system but its extension to all such systems will be manifest. We start with a definition and brief review of the main properties of this model in the standard Poisson bracket formulation. Then we point out that corresponding to the choice of any one of the $2 N-1$ first integrals as the Hamiltonian function there exists a different Poisson bracket description of motion for the rational CalogeroMoser dynamics. Each Poisson tensor obtained in this way is inequivalent to but compatible with all the others. These Poisson tensors cannot be inverted to yield symplectic structure, so here we have the example of an even dimensional dynamical system that admits Poisson but not symplectic structure. We shall present the explicit form of the complete Poisson structures for the rational Calogero-Moser system in terms of the original dynamical variables only for the case of $N=2$ which admits tri-Poisson structure.

The Hamiltonian structure of the rational Calogero-Moser system had earlier been discussed by Magri, Morosi and Ragnisco [7] and Magri and Mursico [8]. Their results and those we shall present in this paper stand in sharp contrast. We present a new alternative to the bi-Hamiltonian approach of these authors.

\section{Rational Calogero-Moser model}

The $N$-particle rational Calogero-Moser model is defined by the Hamiltonian function

$$
H=\frac{1}{2} \sum_{i=1}^{N} p_{i}^{2}+\frac{g^{2}}{2} \sum_{i \neq j}^{N} \frac{1}{\left(q^{i}-q^{j}\right)^{2}}
$$


where $g$ is a coupling constant. The canonical coordinates $q^{i}$ and momenta $p_{j}$ satisfy the standard Poisson bracket relations

$$
\left\{q^{i}, p_{j}\right\}=\delta_{j}^{i} \quad i, j=1, \ldots, N
$$

and we shall find it convenient to refer to the full set of dynamical variables by a collective symbol

$$
x^{A} \in\left\{q^{1}, \ldots, q^{n}, p_{1}, \ldots, p_{n}\right\} \quad A=1, \ldots, 2 N
$$

so that here and in the following capital Latin indices will range over $2 \mathrm{~N}$ values. Then

$$
\left\{x^{A}, x^{B}\right\}=J^{A B}=\left(\begin{array}{cc}
0 & I \\
-I & 0
\end{array}\right)
$$

is the canonical Poisson tensor.

It is the existence of a complete set of $N$ functionally independent and globally defined integrals of motion $I_{k} \quad k=1, \ldots, N$ that makes the rational Calogero-Moser model Liouville integrable. These integrals can be generated from the conserved quantity $I_{N}$, 9]

$$
I_{N} \equiv \exp \left(-\frac{g^{2}}{2} \sum_{i \neq j} \frac{1}{\left(q^{i}-q^{j}\right)^{2}} \frac{\partial^{2}}{\partial p_{i} \partial p_{j}}\right) \prod_{k=1}^{N} p_{k}
$$

by taking its successive Poisson brackets with $\sum_{i=1}^{N} q^{i}$. In this way we obtain the conserved Hamiltonians

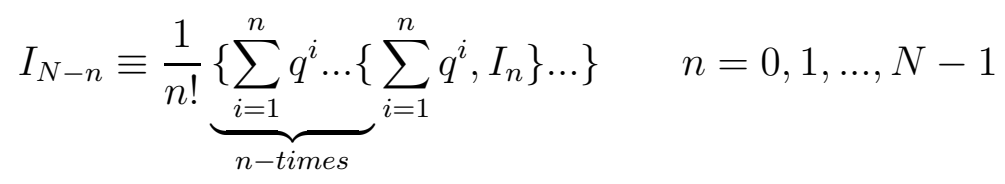

which are in involution with respect to the standard Poisson bracket defined by (4).

Besides these $N$ first integrals of motion the rational Calogero-Moser system admits $N-1$ additional functionally independent integrals of motion $K_{m}$, where $m=2, \ldots, N$ which are defined by the formula 10

$$
K_{m}=m g_{1} I_{m}-g_{m} I_{1}, \quad m=2, \ldots N
$$

where

$$
g_{m}=\frac{1}{2}\left\{\sum_{i=1}^{N}\left(q^{j}\right)^{2}, I_{m}\right\}
$$


with $I_{m}$ given by (6). In general, these super-integrals are not in involution with respect to the canonical Poisson bracket defined by (4).

Just as we found it convenient to use a collective label for coordinates and momenta (3), in what follows it will be useful to introduce a collective label for the set of first integrals $I_{n}$ and super-integrals $K_{m}$ according to

$$
H_{(\alpha)} \in\left\{I_{1}, \ldots, I_{n}, K_{2}, \ldots, K_{N}\right\} \quad \alpha=1, . ., 2 N-1
$$

and use Greek letters exclusively to label the $2 N-1$ conserved Hamiltonians. The parentheses enclosing the Greek indices are there for a cautionary purpose, namely in the formulae that follow, they don't take part in the summation convention.

\section{Poisson structures}

The equations of motion for the rational Calogero-Moser system are given by

$$
\frac{d x^{A}}{d t} \equiv \dot{x}^{A}=J^{A B} \partial_{B} H \equiv J^{A B} \frac{\partial H}{\partial x^{B}}
$$

with the Poisson tensor (4A) and Hamiltonian function (11). If we consider a trajectory $x^{A}=x^{A}(t)$ which is a solution of equations of motion, then we have

$$
\dot{H}_{(\alpha)}=\dot{x}^{K} \partial_{K} H_{(\alpha)}=0
$$

and due to the functional independence of the first integrals $H_{(\alpha)}$, their gradients $\partial_{K} H_{(\alpha)}$ define $2 N-1$ linearly independent vectors orthogonal to the velocity vector. By taking their full cross-product we determine a unique direction at each point in phase space which is precisely that of the velocity vector. Then the trajectory is given by

$$
\dot{x}^{I}=V(x) \varepsilon^{I M_{1} \ldots M_{2 N-1}}\left(\partial_{M_{1}} H_{(1)}\right) \ldots\left(\partial_{M_{2 N-1}} H_{(2 N-1)}\right)
$$

where $\varepsilon^{A_{1} \ldots A_{2 N}}$ with $\varepsilon^{1, \ldots, 2 N}=1$ is the $2 N$-dimensional completely antisymmetric Levi-Civita symbol and $V(x)$ is a factor of proportionality which will be determined by the requirement that both the magnitude as well as the direction of the velocity vector in phase space is given by eq.(12). Then the equations of motion will be identical to those of the rational CalogeroMoser system. This factor of proportionality is time-independent i.e. $V$ is a 
function of integrals of motion $H_{(\alpha)}$ only and plays the role of volume density in phase space. So, more properly, the Levi-Civita tensor density is given by

$$
\epsilon^{A_{1} \ldots A_{2 N}}=\frac{1}{V^{-1}} \varepsilon^{A_{1} \ldots A_{2 N}}
$$

but we shall use it only in the numeric form (12) for ease of manipulation in the calculations that will follow.

The key to the construction of Poisson tensors associated with superintegrable dynamical systems such as the rational Calogero-Moser system is based on the simple observation that eqs.(12) can be written in the form

$$
\dot{x}^{I}=J_{(\alpha)}^{I M_{\alpha}} \partial_{M_{\alpha}} H_{(\alpha)} \quad \alpha=1, \ldots, 2 N-1
$$

where

$$
J_{(\alpha)}^{I M_{\alpha}}=V \varepsilon^{I M_{1} \ldots M_{\alpha} \ldots M_{2 N-1}}\left(\partial_{M_{1}} H_{(1)}\right) \ldots\left(\partial_{M_{\alpha} H}(\alpha)\right) \ldots\left(\partial_{M_{2 N-1}} H_{(2 N-1)}\right)
$$

and a hat over a quantity indicates that it should be omitted. Therefore, in eqs.(14) we have the expression for $2 N-1$ skew-symmetric tensors which yield the equations of motion of the rational Calogero-Moser system in the Poisson bracket form (13). Of course to claim that in eqs. (14) we have the definition of Poisson tensors that define Poisson brackets

$$
\{f, g\}_{(\alpha)} \equiv J_{(\alpha)}^{K L}\left(\partial_{K} f\right)\left(\partial_{L} g\right)
$$

requires proof that the Jacobi identities are satisfied. Furthermore if the Jacobi identities are satisfied for a linear combination of all $2 N-1$ skewsymmetric tensors given by (14), then we have a Poisson pencil. In order to show that $J_{(\alpha)}^{A B}$ given by eqs.(14) define Poisson tensors we must prove skew-symmetry and Jacobi identities

$$
J_{(\alpha)}^{K[C} \partial_{K} J_{(\alpha)}^{A B]}=0
$$

where square brackets denote complete skew-symmetrization. The former is obvious and in order to prove the latter we use the identity

$$
\varepsilon^{K M_{2} \ldots M_{2 N-1}\left[C_{\varepsilon}\right.} \varepsilon^{A B] P_{2} \ldots P_{2 N-1}}=\frac{2}{(2 N) !} \sum_{i=2}^{2 N-1} \varepsilon^{K P_{i} M_{2} \ldots M_{2 N-1}} \varepsilon^{P_{2} \ldots P_{i-1} C p_{i+1} \ldots P_{2 N-1} B A}
$$


satisfied by the Levi-Civita tensor. This identity is a general result for the Levi-Civita tensor which is particularly useful for complete skew-symmetrization in the indices $A, B, C$ in eq.(15). There is no particular distinction for different values of $\alpha$ in eqs.(14) for $J_{\alpha}^{K M}$ and therefore without loss of generality we can put $\alpha=1$ in proving the Jacobi identities (15). Then using the definition (14) and the identity (16) we find that

$$
\begin{aligned}
& \varepsilon^{K M_{2} \ldots M_{2 N-1}[C} \varepsilon^{A B] P_{2} \ldots P_{2 N-1}}\left(V^{2} \partial_{K}+V \partial_{K} V\right) \\
& {\left[\left(\partial_{M_{2}} H_{(2)}\right) \ldots\left(\partial_{M_{2 N-1}} H_{(2 N-1)}\right)\left(\partial_{P_{2}} H_{(2)}\right) \ldots\left(\partial_{P_{2 N-1}} H_{(2 N-1)}\right)\right] } \\
= & \frac{2}{(2 N) !} \sum_{I=2}^{2 N-1} \varepsilon^{K P_{i} M_{2} \ldots M_{2 N-1}} \varepsilon^{P_{2} \ldots C \ldots P_{2 N-1} B A}\left(V^{2} \partial_{K}+V \partial_{K} V\right) \\
& {\left[\left(\partial_{M_{2}} H_{(2)}\right) \ldots\left(\partial_{M_{2 N-1}} H_{(2 N-1)}\right)\left(\partial_{P_{2}} H_{(2)}\right) \ldots\left(\partial_{P_{2 N-1}} H_{(2 N-1)}\right)\right] }
\end{aligned}
$$

where the third line above is anti-symmetric in the indices $P_{i}, M_{i}$ but the expression it is contracted with in the last line above is symmetric in these indices. Thus we have established that the right hand side of eq.(17) vanishes and consequently its left hand side, which is just the Jacobi identity (15), must also vanish.

\subsection{Properties of these Poisson structures}

The compatibility condition for Poisson tensors requires that for any $\alpha, \beta=$ $1, \ldots 2 N-1$ and $\Lambda \in R$ the linear combination $\{\cdot, \cdot\}_{(\alpha)}+\Lambda\{\cdot, \cdot\}_{(\beta)}$ satisfies the Jacobi identify, thus forming a Poisson pencil. This compatibility condition is just

$$
J_{\alpha}^{K[C} \partial_{K} J_{\beta}^{A B]}+J_{\beta}^{K[C} \partial_{K} J_{\alpha}^{A B]}=0
$$

and its proof proceeds in the same way as the Jacobi identity. Using the identity (16) we split (18) into left and right hand sides as in eq.(17) and show that the right hand side vanishes.

The general construction of the Poisson tensors for super-integrable systems that we gave in section 3 immediately leads to the fact that they are all degenerate

$$
\operatorname{det}\left|J_{(\alpha)}^{A B}\right|=\varepsilon_{A_{1} A_{2} \ldots A_{2 N}} \varepsilon_{B_{1} B_{2} \ldots B_{2 N}} J_{(\alpha)}^{A_{1} B_{1}} J_{(\alpha)}^{A_{2} B_{2}} \ldots J_{(\alpha)}^{A_{2 N} B_{2 N}}=0
$$

and consequently there exists no symplectic structure corresponding to any one of the Poisson structures (14). 


\subsection{Note added}

One of the referees of the first version of this paper has pointed out that we can take the first integrals $H_{1}, H_{2}, \ldots, H_{2 N-1}$ together with $H_{2 N}$ satisfying

$$
\dot{H}_{2 N}=1
$$

as new coordinates in phase space so that these Poisson structures assume simply the Darboux form. Then we have

$$
\begin{aligned}
\left\{H_{\alpha}, H_{2 N}\right\}_{\beta} & =\delta_{\alpha \beta} \\
\left\{H_{\alpha}, H_{\beta}\right\}_{\gamma} & =0 .
\end{aligned}
$$

This is always possible locally and in particular we can take

$$
H_{2 N}=\frac{\sum_{i=1}^{N} q^{i}}{\sum_{i=1}^{N} p_{i}}
$$

but there are many other candidates for $H_{2 N}$. Here we also see that $V$ plays the role of the inverse of Jacobian and the fact that it depends only on $H_{1}, \ldots, H_{2 N-1}$ can be traced back to Liouville theorem for original dynamics. The statement (21) is a very nice compact way to remember the $2 N-1$ Poisson structures.

One can check that this structure coincides locally with the one given in terms of original phase space coordinates by eq.(13). However, the equivalence holds only locally as long as one is able to express original coordinates $x^{A}$ as well-defined unique functions of first integrals. But according to implicit function theorem this is possible only locally. On the other hand at the heart of the notion of (super-)integrability lies the requirement of globality, without it any time-independent Hamiltonian system is, locally, (super-)integrable.

\section{Remarks}

We have presented a general framework constructing the Poisson structure of super-integrable systems. It is in a sense an extension of Nambu's construction of the Poisson structure for the free Euler top [5]. We have presented the details for the rational Calogero-Moser system but it is obvious that this construction can be repeated for any integrable system which admits the 
maximal number of first integrals of motion. There are several important issues raised by this construction.

First, the standard Poisson tensor (4) is not among the $J_{(\alpha)}^{K M}$ structures given by eq.(14).

Each Poisson tensor $J_{(\alpha)}^{A B}$ that generates the Calogero-Moser dynamics through the Hamiltonian function $H_{(\alpha)}$ admits $2(N-1)$ Casimirs

$$
J_{(\alpha)}^{A B} \partial_{B} H_{(\beta)}=0 \quad \beta \neq \alpha
$$

which consist of all the remaining integrals of motion. Consequently the Calogero-Moser system admits no higher flows and in this Poisson structure there exists no recursion operator. As a matter of fact there is no need for the recursion operator because we start with the full set of integrals of motion from the very beginning.

Neither one of our Poisson tensors is compatible with the standard one given by eq.(4).

The Poisson tensors $J_{(\alpha)}^{A B}$ are degenerate for all $\alpha$ and there exists no symplectic 2-form.

Finally, we note that we can summarize the whole Poisson structure of the Calogero-Moser system by writing a single expression

$$
J^{A B}=\sum_{\beta=1}^{2 N-1} J_{(\beta)}^{A B} \quad \alpha=1,2, \ldots, 2 N-1
$$

and the equations of motion (10) can be written in the form

$$
\dot{x}^{A}=J^{A B} \partial_{M} H_{(\alpha)}
$$

for all $\alpha$ because except for the $\alpha^{\text {th }}$ term, $H_{\alpha}$ is a Casimir for each term in the sum (24).

The results we have presented above for the complete Poisson structure of the general rational Calogero-Moser system stand in sharp contrast to the bi-Hamiltonian structure of this system given in [7] and [8]. There is an important lesson to be drawn from this discussion. Namely, there exists alternative avenues to constructing the Poisson structure of completely integrable dynamical systems. 


\section{Appendix}

We shall now give explicit expressions for our construction of Poisson tensors in terms of the original canonical coordinates and momenta for the simplest

non-trivial rational Calogero-Moser system. This is the two particle, $N=2$ system that admits tri-Poisson structure. First we list the conserved Hamiltonians

$$
\begin{aligned}
H_{1} & =p_{1}+p_{2} \\
H_{2} & =\frac{1}{2}\left(p_{1}^{2}+p_{2}^{2}\right)+\frac{g^{2}}{2\left(q^{1}-q^{2}\right)^{2}} \\
H_{3} & =2\left(q^{1}+q^{2}\right) H_{2}-\left(p_{1} q^{1}+p_{2} q^{2}\right) H_{1} \\
& =p_{1}^{2} q_{2}+p_{2}^{2} q^{1}-p_{1} p_{2}\left(q^{1}+q^{2}\right)+\frac{g^{2}\left(q^{1}+q^{2}\right)}{\left(q^{1}-q^{2}\right)^{2}}
\end{aligned}
$$

that will go into the construction (14). Then for the first Poisson tensor we find

$$
\begin{aligned}
J_{1}^{12} & =\frac{g^{2}}{\left(q^{2}-q^{1}\right)^{3}} \\
J_{1}^{13} & =\frac{1}{2 H_{2}^{\prime}}\left[\left(p_{1}-p_{2}\right) p_{1} p_{2}+g^{2}\left(p_{1}+p_{2}\right) \frac{q^{1}+q^{2}}{\left(q^{1}-q^{2}\right)^{3}}\right] \\
J_{1}^{14} & =\frac{1}{2 H_{2}^{\prime}}\left\{\left(p_{1}-p_{2}\right) p_{2}^{2}+\frac{g^{2}}{\left(q^{1}-q^{2}\right)^{3}}\left[p_{1}\left(q^{1}+q^{2}\right)-p_{2}\left(q^{1}-3 q^{2}\right)\right]\right\} \\
J_{1}^{23} & =\frac{1}{2 H_{2}^{\prime}}\left\{\left(p_{2}-p_{1}\right) p_{1}^{2}-\frac{g^{2}}{\left(q^{1}-q^{2}\right)^{3}}\left[p_{1}\left(3 q^{1}-q^{2}\right)+p_{2}\left(q^{1}+q^{2}\right)\right]\right\} \\
J_{1}^{24} & =\frac{1}{2 H_{2}^{\prime}}\left[\left(p_{2}-p_{1}\right) p_{1} p_{2}-\left(p_{1}+p_{2}\right) g^{2} \frac{q^{1}+q^{2}}{\left(q^{1}-q^{2}\right)^{3}}\right] \\
J_{1}^{34} & =\frac{1}{2 H_{2}^{\prime}}\left(p_{1}-p_{2}\right)^{2}\left(q^{1}-q^{2}\right)
\end{aligned}
$$

where

$$
H_{2}^{\prime}=\frac{1}{2}\left(p_{1}-p_{2}\right)^{2}+\frac{g^{2}}{\left(q^{1}-q^{2}\right)^{2}}
$$

is a conserved quantity and $V^{-1}=2 H_{2}^{\prime}$ serves as the volume density in phase space. The components of the second Poisson tensor are given by

$$
J_{2}^{12}=0
$$




$$
\begin{aligned}
J_{2}^{13} & =\frac{1}{2 H_{2}^{\prime}}\left[\left(p_{2}-p_{1}\right) p_{1}-g^{2} \frac{3 q^{1}+q^{2}}{\left(q^{1}-q^{2}\right)^{3}}\right] \\
J_{2}^{14} & =\frac{1}{2 H_{2}^{\prime}}\left[\left(p_{2}-p_{1}\right) p_{2}-g^{2} \frac{q^{1}+3 q^{2}}{\left(q^{1}-q^{2}\right)^{3}}\right] \\
J_{2}^{23} & =\frac{1}{2 H_{2}^{\prime}}\left[\left(p_{1}-p_{2}\right) p_{1}+g^{2} \frac{3 q^{1}+q^{2}}{\left(q^{1}-q^{2}\right)^{3}}\right] \\
J_{2}^{24} & =\frac{1}{2 H_{2}^{\prime}}\left[\left(p_{1} p_{2}\right) p_{2}+g^{2} \frac{q^{1}+3 q^{2}}{\left(q^{1}-q^{2}\right)^{3}}\right] \\
J_{2}^{34} & =\frac{1}{2 H_{2}^{\prime}}\left[-p_{1}\left(q^{1}+3 q^{2}\right)+p_{2}\left(3 q^{1}+q^{2}\right)\right]
\end{aligned}
$$

and the third one is the simplest

$$
J_{3}=\frac{1}{2 H_{2}^{\prime}}\left(\begin{array}{cccc}
0 & 0 & \frac{g^{2}}{\left(q^{1}-q^{2}\right)^{3}} & \frac{g^{2}}{\left(q^{1}-q^{2}\right)^{3}} \\
0 & 0 & \frac{g^{2}}{\left(q^{2}-q^{1}\right)^{3}} & \frac{g^{2}}{\left(q^{2}-q^{1}\right)^{3}} \\
\frac{g^{2}}{\left(q^{2}-q^{1}\right)^{3}} & \frac{g^{2}}{\left(q^{1}-q^{2}\right)^{3}} & 0 & p_{1}-p_{2} \\
\frac{g^{2}}{\left(q^{2}-q^{1}\right)^{3}} & \frac{g^{2}}{\left(q^{1}-q^{2}\right)^{3}} & p_{2}-p_{1} & 0
\end{array}\right) .
$$

It can be directly verified that they form a Poisson pencil, namely

$$
\mathcal{J}=\sum_{i=1}^{3} c_{i} J_{i}
$$

where $c_{i}$ are arbitrary constants satisfies the Jacobi identities (15).

\section{Acknowledgement}

We thank the referee for his remarks reported in section 3.2. C.G. would like to thank Piotr Kosinski for very useful comments and discussions.

\section{References}

[1] F.Magri, J. Math. Phys. 19 (1978) 1156

F. Magri, in Nonlinear Evolution Equations and Dynamical Systems, 
editors Lecture Notes in Phys. 120, Springer, New York (1980) p. 233

F. Magri, C. Morosi and G. Tondo, Comm. Math. Phys. 115 (1988) 457

G. Landi, G. Marmo and G. Vilasi, J. Math. Phys. 35 (1994) 808

[2] F. Calogero, J. Math. Phys. 10 (1969) 2191

F. Calogero, J. Math. Phys. 12 (1971) 419 (ibid 37 (1996) 3646)

F. Calogero, Lett. Nuovo Cimento 13 (1975) 411

F. Calogero and G. Marchioro, J. Math. Phys. 15 (1974) 1425

B. Sutherland, J. Math. Phys. 12 (1971) 246, 251

B. Sutherland, Phys. Rev. 14 (1971) 2019

J. Moser, Adv. Math. 16 (1975) 1

M. A. Olshanetsky and A. M. Perelomov, Phys. Rep. 71 (1981) 313

M. A. Olshanetsky and A. M. Perelomov, Phys. Rev. 94 (1983) 313

S. Wojciechowski, Phys. Lett. A 95 (1998) 272

C. Gonera, J. Phys. A 31 (1998) 4465

[3] S. N. M. Ruijsenaars, H. Schneider Ann. Phys. (NY) 170 (1986) 370

S. N. M. Ruijsenaars, Comm. Math. Phys. 110 (1987) 191

[4] N. W. Evans, Phys. Lett. A 147 (1990) 483

[5] Y. Nambu, Phys. Rev. D 7 (1973) 2405

[6] H. Gümral and Y. Nutku, J. Math. Phys. 34 (1993) 5691

[7] F. Magri, C. Morosi and O. Ragnisco Comm. Math. Phys. 99 (1985) 115

[8] F. Magri "Some developements of the concept of Poisson manifold in the sense of Lichnerowicz" in Gravitation, Electromagnetism and Geometrical Structures G. Ferrarese editor, Pitagne Edifice Bologna p. 207

[9] K. Sawada and T. Kotera J. Phys. Soc. Japan. 39 (1975) 1614

[10] C. Gonera, Phys. Lett. A 237 (1998) 365 